\title{
CLINICAL IMPLEMENTATION OF INTENSITY-MODULATED ARC THERAPY (IMAT) FOR RECTAL CANCER
}

\author{
Wim Duthoy, M.D., * Werner De Gersem, Ir., ${ }^{*}$ Koen Vergote, M.Sc., ${ }^{*}$ \\ Tom Boterberg, M.D., Ph.D., ${ }^{*}$ Cristina Derie, G.N., ${ }^{*}$ Peter Smeets, M.D., $\dagger$ \\ Carlos De Wagter, Ir., Ph.D.,* and Wilfried De Neve, M.D., Ph.D.* \\ Departments of * Radiotherapy and ${ }^{\dagger}$ Radiology, Ghent University Hospital, Ghent, Belgium
}

\begin{abstract}
Purpose: In rectal cancer, combined radiotherapy and chemotherapy, either pre- or postoperatively, is an accepted treatment. Late small bowel (SB) toxicity is a feared side effect and limits radiation-dose escalation in a volume-dependent way. A planning strategy for intensity- modulated arc therapy (IMAT) was developed, and IMAT was clinically implemented with the aim to reduce the volume of SB irradiated at high doses and thus reduce SB toxicity. We report on the treatment plans of the first 7 patients, on the comparison of IMAT with conventional 3D planning (3D), and on the feasibility of IMAT delivery.

Methods and Materials: Seven patients, who were referred to our department for preoperative $(n=4)$ or postoperative $(n=3)$ radiotherapy for rectal cancer, gave written consent for IMAT treatment. All patients had a planning CT in prone position. The delineation of the clinical target volume was done after fusion of CT and MRI, with the help of a radiologist. For the IMAT plan, arcs were generated using an anatomybased segmentation tool. The optimization of the arcs was done by weight optimization (WO) and leaf position optimization (LPO), both of which were adapted for IMAT purposes. The 3D plans used one posterior and two lateral wedged beams, of which the outlines were shaped to the beam's-eye view projection of the planning target volume (PTV). Beam WO was done by constrained matrix inversion. For dose-volume histogram analysis, all plans were normalized to 45 Gy as median PTV dose. Polymer gel dosimetry (PGD) on a humanoid phantom was used for the validation of the total chain (planning to delivery). IMAT treatments were delivered by an Elekta SliPlus linear accelerator using prototype software with the same interlock class as in clinical mode.

Results: The IMAT plan resulted in 3 to 6 arcs, with a mean delivery time of $6.3 \mathrm{~min}$ and a mean of 456 monitor units (MU) for a 180 cGy fraction. The minimal dose in the PTV was not significantly different between 3D and IMAT plans. Inhomogeneity was highest for the IMAT plans (14.1\%) and lowest for the 3D plans (9.9\%). Mean dose to the SB was significantly lower for the IMAT plans (12.4 Gy) than for the 3D plans (17.0 Gy). The volume of SB receiving less than any dose level was lower for the IMAT plans than for 3D plans. Integral dose was lower in the IMAT plans than for the 3D plans (respectively $244 \mathrm{~J}$ and $262 \mathrm{~J}$ to deliver $45 \mathrm{~Gy}$ ). Differences between the PGD measured dose and the calculated dose were as small for IMAT as for 3D treatments.

Conclusion: IMAT plans are deliverable within a 5-10-minute time slot, and result in a lower dose to the SB than 3D plans, without creating significant underdosages in the PTV. PGD showed that IMAT delivery is as accurate as 3D delivery. () 2004 Elsevier Inc.
\end{abstract}

Intensity-modulated arc therapy (IMAT), Rectal cancer, Small bowel toxicity.

\section{INTRODUCTION}

The use of radiotherapy, either preoperatively or postoperatively, in addition to systemic chemotherapy, decreases the local recurrence rate and improves survival in rectal cancer
(1). Randomized trials of preoperative radiation and surgery vs. surgery alone showed a radiation-dose dependent reduction of local failure in the combined modality arm, with a halving of the local recurrence rate at biologically effective doses of $\geq 30$ Gy (2). Preoperative radiation therapy to a
Reprint requests to: Wim Duthoy, M.D., Division of Radiotherapy, Ghent University Hospital, De Pintelaan 185, 9000 Ghent, Belgium. Tel: (+32) 92403074. Fax: (+32) 92403863; E-mail: wimd@krtkg1.UGent.be

The project "Conformal Radiotherapy Ghent University Hospital" is supported by the Belgische Federatie tegen Kanker and by grants from the Fonds voor Wetenschappelijk Onderzoek (FWO) Vlaanderen (G.0183.03), the University of Ghent (GOA 12050401, BOF 01112300, 011VO497, 011F1700), and the Cen- trum voor Studie en Behandeling van Gezwelziekten. Wim Duthoy is a Research Assistant (Aspirant) of the FWO.

Acknowledgments-Jan Schauvliege from Elekta is acknowledged for fine-tuning the linear accelerator for IMAT purposes. Nucletron is acknowledged for providing the Oncentra Treatment Planning System.

Received Jan 20, 2004, and in revised form Mar 16, 2004. Accepted for publication Apr 2, 2004. 
Table 1. Patient and planning description*

\begin{tabular}{|c|c|c|c|c|c|c|c|c|c|}
\hline $\begin{array}{l}\text { Patient } \\
\text { No. }\end{array}$ & $\begin{array}{c}\mathrm{RT} \\
\text { timing }\end{array}$ & TNM & $\mathrm{Vol}_{\mathrm{sb}_{3 \mathrm{D}}}(\mathrm{cc})$ & $\begin{array}{c}\# \\
\operatorname{arcs} \\
\end{array}$ & $\begin{array}{c}\# \\
\text { MUs }\end{array}$ & $\begin{array}{c}\mathrm{CDT} \\
(\text { mean } \pm \mathrm{SD})\end{array}$ & DRDT & $\begin{array}{c}\text { \# MUs } \\
\text { (W/UW) }\end{array}$ & DRDT \\
\hline 1 & pre & cT3 cN1 & 234.4 & 5 & 526 & $5.8 \pm 0.4$ & 5.4 & $389 / 109$ & 2.8 \\
\hline 2 & pre & $\mathrm{cT} 2 \mathrm{cN} 1$ & 29.7 & 6 & 508 & $9.4 \pm 0.3$ & 7.6 & $350 / 87$ & 2.4 \\
\hline 3 & post & pT3 pNo & 22.6 & 6 & 421 & $7.5 \pm 0.3$ & 6.3 & $302 / 92$ & 2.3 \\
\hline 6 & pre & cT3 cN1 & 0.8 & 5 & 392 & $5.8 \pm 0.1$ & 5.5 & $363 / 92$ & 2.4 \\
\hline 7 & post & pT3 pN1 & 146.3 & 4 & 337 & $5.6 \pm 0.7$ & 4.8 & $211 / 144$ & 2.6 \\
\hline
\end{tabular}

Abbreviations: $\mathrm{Vol}_{\mathrm{sb}_{3 \mathrm{D}}}=$ the volume of small bowel receiving more than $90 \%$ of the target prescription dose in the $3 \mathrm{D}$ plan; $\mathrm{IMAT}=$ intensity-modulated arc therapy.

* The 7 patients are ordered chronologically. RT timing is the order of radiotherapy in relation to the surgery, in which "pre" stands for preoperative radiotherapy, and "post" for postoperative radiotherapy.

For IMAT the number of arcs is given, as is the total number of monitor units (MUs) for a fraction of 180 cGy. Clinical delivery time (CDT) and dummy run delivery time (DRDT) is given. All time measurements are given in minutes. For the 3D plans, MUs are split up into the wedged (W) and unwedged (UW) part.

dose of 40-45 Gy in 1.8-2 Gy per fraction, usually combined with 5-fluorouracil-based chemotherapy is the standard schedule in many European centers.

Although some reports suggest further improvement in local control for higher doses, especially in high-stage rectal cancer $(3,4)$, acute and chronic small bowel (SB) and rectal toxicity (5) seem to limit further dose escalation. For small bowel, reported incidences of acute Grade III-IV diarrhea vary between $15 \%$ and $40 \%$, depending on grading systems, type of surgery, administration of adjuvant chemotherapy, and other variables such as comorbidity $(6,7)$. Chronic SB radiation toxicity is irreversible and can be life-threatening. Actuarial 5-year estimates of severe chronic SB toxicity vary between $7 \%$ and $42 \%(8,9)$ at doses of $45-50 \mathrm{~Gy}$. The incidence of both acute and late SB toxicity is related to the volume of SB in the treatment fields $(6,7,9)$. Several possible methods have been examined for minimizing the portion of SB in the treatment field. For a review of these different techniques, the reader is referred to Letschert (10). A new possibility to reduce the volume of SB irradiated to high doses consists of intensity modulation (IM). IM offers the possibility to spare an organ at risk (OAR) lying in the concavity of a planning target volume (PTV). This anatomic relationship occurs in rectal cancer, where SB is partially surrounded by the clinical target volume (CTV), consisting of the rectum and its draining lymph node regions. Because of the large internal radius of the concave PTV in rectal cancer, a large number of beam directions may be favorable (11). With arc therapy, the number of beam directions is infinite, and thus intensity-modulated arc therapy (IMAT) $(11,12)$ might be a logical approach to this clinical problem. The goals of this study are as follows: (1) to present our planning strategy and first experience of IMAT for rectal cancer; (2) to report the results of a planning comparison between conventional radiotherapy (3D) and IMAT; (3) to validate the IMAT delivery method using polymer gel dosimetry.

\section{METHODS AND MATERIALS}

\section{Delineation}

Between February 2003 and November 2003, 7 patients with a histologically proven adenocarcinoma of the rectum were treated with IMAT. All patients signed an informed consent for inclusion in this feasibility study, approved by the local ethics committee. Some clinical data of these patients are shown in Table 1 A planning computed tomography (CT) was performed with the patient in prone position. For Patients 5, 6, and 7, a bellyboard was used (Sinmed, Reeuwijk, The Netherlands). CT slice thickness was $0.3 \mathrm{~cm}$, and the scanned volume extended from the third lumbar vertebra down to $10 \mathrm{~cm}$ caudal to the obturator foramina. No contrast enhancement was used. No attempts were made to reduce variation of bladder filling. All patients underwent diagnostic magnetic resonance imaging (MRI), which was electronically fused with the planning CT. This was done using the landmark method of the Oncentra Treatment Planning System (Nucletron B.V., Veenendaal, The Netherlands). In those patients who were sent for preoperative radiotherapy, the gross tumor volume (GTV) was delineated using the fused CT-MRI. The fused CT-MRI was also used for the delineation of all other target subvolumes. These included the following structures: rectum, mesorectal space, presacral space and the lymph node regions along the common iliac (up to and including the level of the first sacral vertebra), the internal iliac, the superior rectal, and the internal obturator vessels. In patients with a tumor lying $6 \mathrm{~cm}$ or less from the anal verge, the perineal area was also included. The contouring of these target subvolumes was done together with a radiologist (P.S.). Because the internal motion of the different target subvolumes is different (13), the rectum was expanded anisotropically with $1.5 \mathrm{~cm}$ in the anteroposterior direction, and with $0.5 \mathrm{~cm}$ in the craniocaudal and lateral directions. All other subvolumes were expanded isotropically with $0.5 \mathrm{~cm}$. All unexpanded subvol- 


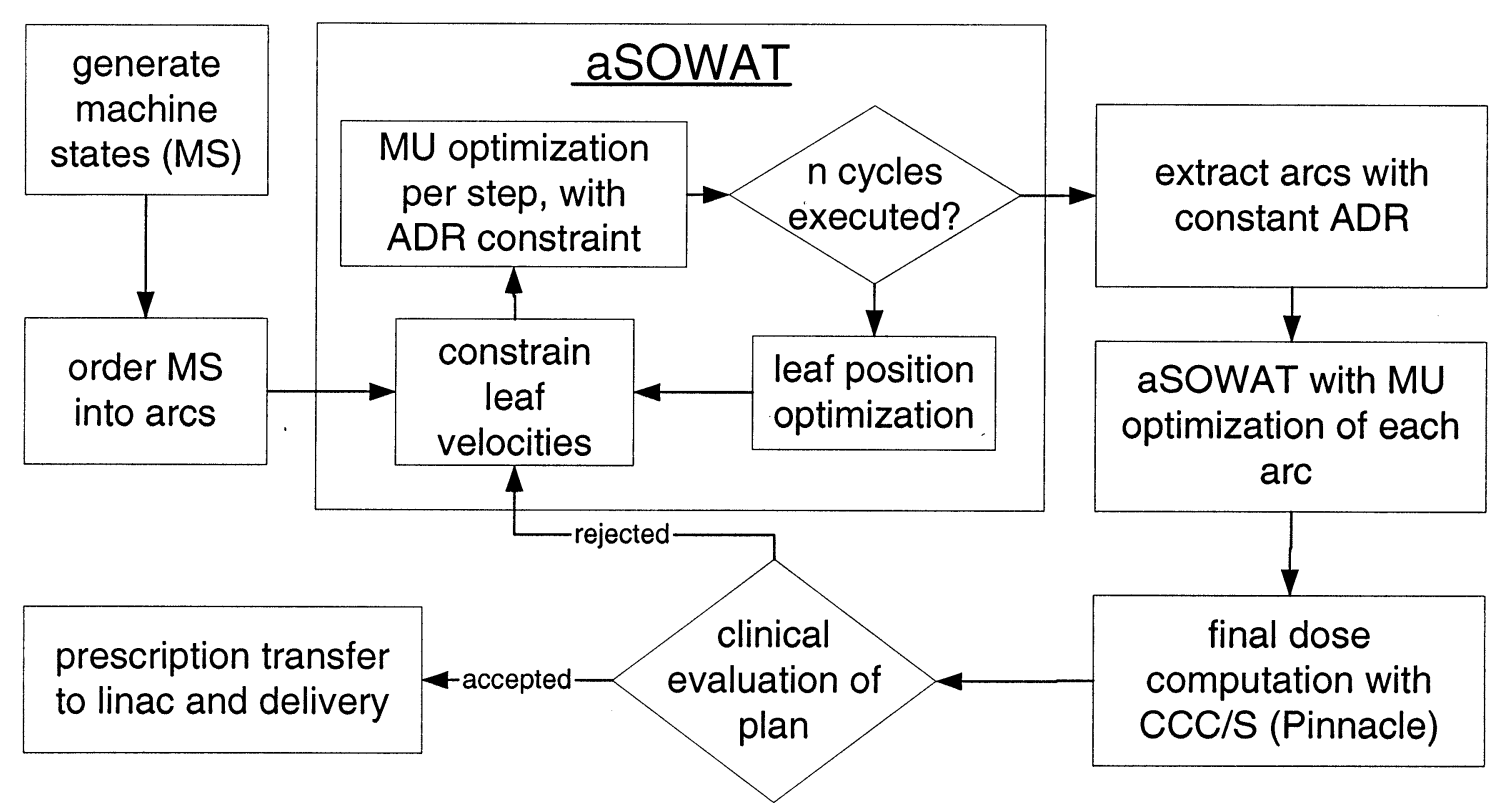

Fig 1. Intensity-modulated arc therapy (IMAT) planning flowchart. Abbreviations: ADR = angular delivery rate; aSOWAT $=$ arc-therapy modification of segment outline and weight adaptation tool; $\mathrm{CCC} / \mathrm{S}=$ collapsed cone convolution/superposition calculation algorithm; $\mathrm{MS}=$ machine state. A MS is described by a set of machine parameters that uniquely define beam direction, aperture, and photon beam quality. After MS generation, MSs of the same class are then ordered into arcs. These arcs are given as input to aSOWAT. This is our optimization tool, which was adapted for IMAT purposes. aSOWAT starts with a check of the leaf velocities in the transition from one MS to the next. If necessary, leaf positions are adapted to meet this constraint. Then, the actual optimization starts with the optimization of the monitor units (MU) per step, in which a step is defined as the transition from one MS to the next. A factor is included in the biophysical cost function to restrict the MU per degree variation (ADR constraint). When a user-defined number of cycles have been executed, aSOWAT is terminated, and the new MS outlines are saved. This is followed by the interactive extraction of deliverable arcs with constant ADR. After all deliverable arcs have been extracted, these are given as input to aSOWAT, but with optimization of the total number of MUs per arc instead of per step. Finally, a dose calculation with the CCC/S algorithm is done, and the plan is evaluated by the clinical staff.

umes were summed to form the CTV, whereas all expanded subvolumes were summed to the PTV.

The OARs that were contoured were bladder and SB. All SB loops were delineated individually up to the CT slice through the middle of the fourth lumbar vertebra. Structures provided to the optimization algorithm included the PTV, bladder, SB, and three structures generated to aid the optimization towards a desirable solution. A first optimization aid structure was made by subtracting the PTV and an additional margin of $0.6 \mathrm{~cm}$ from a $1-\mathrm{cm}$ expansion of the SB, resulting in the structure "small_bowel_exp_optim". Other optimization aid structures were rim_2_cm (= all tissue between 0 and $2 \mathrm{~cm}$ around the PTV) and surr (= all tissue more than $2 \mathrm{~cm}$ from the PTV). For a discussion about the use of these optimization aid volumes, the reader is referred to De Neve et al. (14). The anatomy-based segmentation tool (ABST) (15) needs an OAR of which the projection on the isocentric plane is larger than the PTV projection in the direction perpendicular to the direction of the leaf motion. For this reason, a segmentation structure (segm_str) was made, consisting of SB and bladder, elongated in the craniocaudal direction when necessary. Per patient, the same target structures and OARs were used for both plans.

\section{IMAT planning procedure}

The IMAT planning procedure has been described in more detail previously (11) and is summarized in Fig. 1. Machine states (MSs) are generated using ABST every $8^{\circ}$ within the deliverable range. This deliverable range consists of beam directions in which the radiation beam does not traverse metal components of the couch before entering the patient. In the case of the Elekta SLiPlus couch, the largest deliverable range is obtained by positioning the metal Carms of the couch on $120^{\circ}$ or $150^{\circ}$, as measured from their lateral position. Considering the relative position of the isocenter to the table and the PTV geometry, possible gantry angles ranged from $-136^{\circ}$ to $136^{\circ}$. Thus, no arcs were irradiating the patient from below the couch (anterior to the patient). The ABST resulted in (maximally) four classes of MSs per beam direction, differing from each other only in the coverage of the PTV (Fig. 2). All adjacent MSs of the same class were sorted based on the gantry angle to form an arc (Fig. 2), thus resulting in four arcs. These initial arcs were the input of the optimization, which is done by our in-house developed segment outline and weight adaptation tool (SOWAT), modified for IMAT purposes (aSOWAT) (Fig. 1). aSOWAT optimizes the leaf positions of each MS 


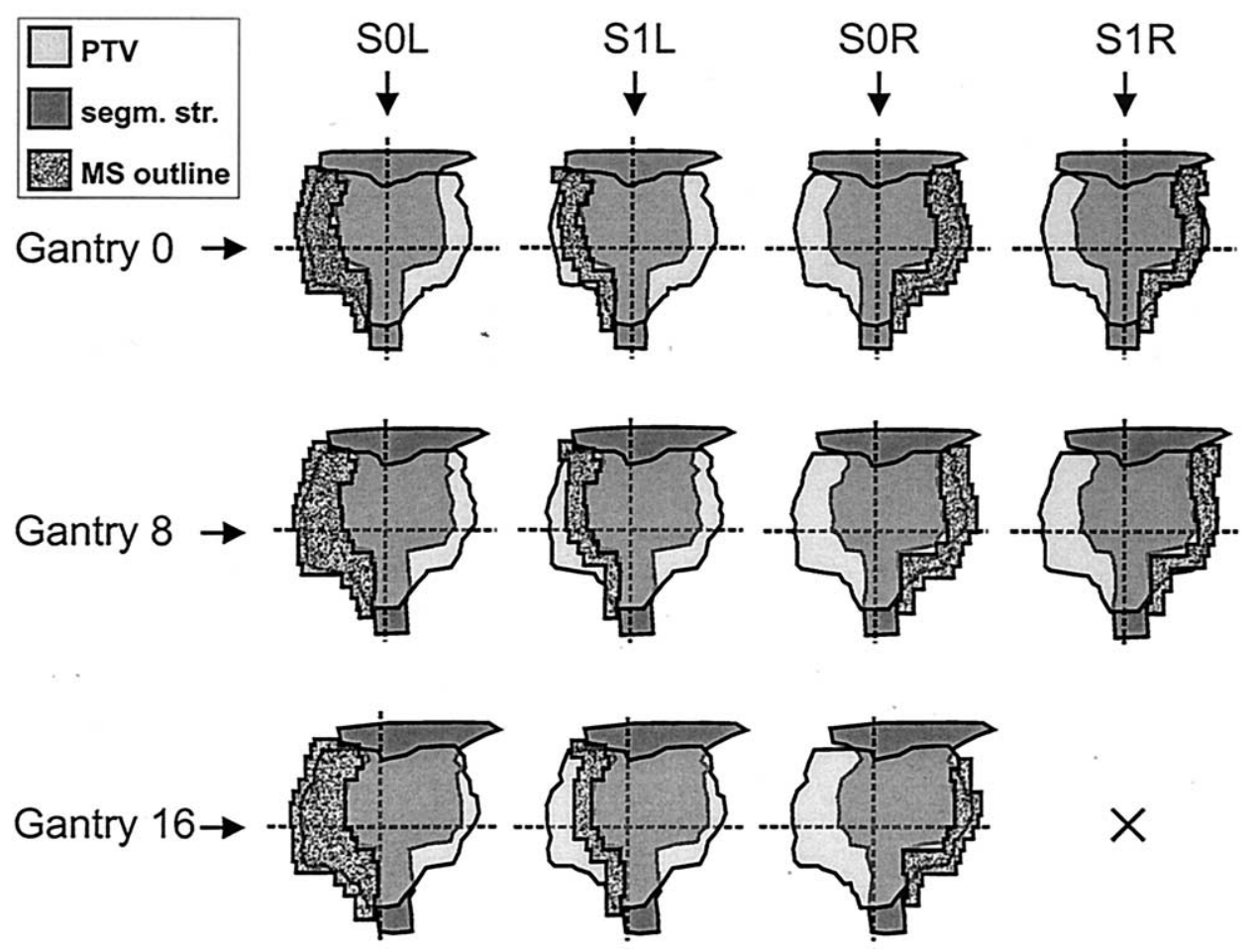

Fig. 2. Anatomy-based arc generation shown in beam's-eye view (BEV). Abbreviations: PTV = planning target volume; Segm. str. = segmentation structure; MS = machine state; SOL = MS covering the total BEV projection of the PTV passing at the left side of the segmentation structure; S1L = MS covering a 2-cm-wide area of the PTV at the left side of the segmentation structure; SOR = MS covering the total BEV projection of the PTV passing at the right side of the segmentation structure. S1R = MS covering a 2-cm-wide area of the PTV at the right side of the segmentation structure. The ABST-generated machine states are shown for three adjacent beam directions (gantry angles $0^{\circ}, 8^{\circ}$, and $16^{\circ}$ ). The horizontal position shows how the different classes (S0L, S1L, S0R, and S1R) were generated per gantry angle. In the vertical direction, the ordering per arc can be appreciated. As can be seen, the BEV projection of the PTV and the segmentation structure does not differ much when the gantry is rotated over $8^{\circ}$. The S1R MS for the gantry on $16^{\circ}$ was not generated, because the width of the S0R MS was less than the user-defined $2 \mathrm{~cm}$ for the S1R MS. This means that the S1R arc will end at a gantry angle of $8^{\circ}$.

and the monitor unit (MU) values of each step, in which a step is defined as the transition from one MS to the next MS. Additional to the previously described IMAT planning strategy, a factor has been added to the objective function to obtain a fluent angular delivery rate (ADR). This factor takes into account the first and second derivative of the ADR, approximated using the first and second finite differences of the MU values of each step. This first aSOWAT optimization results in arcs with a nonconstant ADR, further called virtual arcs, which are presently undeliverable on the Elekta SLiPlus linac. These virtual arcs, however, help to select the start and stop gantry angles of the final, deliverable arcs, for which the ADR has to be constant. The fluently modulating ADR facilitates this extraction of deliverable arcs. To obtain a time-efficient treatment, the number of arcs has to be kept low. Consequently, a one-step extraction of deliverable arcs would impose a very rough discretization of the optimized ADR.

A more efficient procedure was implemented as follows: each arc is separately (and manually) extracted from the ADR graphs displayed on the planning computer. After each extraction of a deliverable arc, the MUs of all deliv- erable arcs are reoptimized, together with the remnant ADR of the virtual arcs. During this short optimization, a factor included in the objective function favors the MUs of the deliverable arcs with respect to the ADR of the virtual arcs. Each extraction transfers MUs from the virtual to the deliverable arcs. By downforcing the remnant ADR, only the important parts of the virtual arcs remain to be extracted in the next deliverable arcs. When the remnant ADR falls to zero by optimization, the extraction procedure is finished. The plan is finalized by an aSOWAT optimization cycle, consisting of optimization of the leaf positions of the final arcs, and optimization of the ADR per (extracted) arc instead of per step, which is equivalent to optimization of the total MU-count of an arc (to be delivered at a constant ADR). A final dose computation was done for $18 \mathrm{MV}$ using the collapsed cone convolution/superposition algorithm from Pinnacle (Philips Medical Systems, Best, The Netherlands), which was used for all (IMAT and 3D) plans. Dose distributions of arcs were approximated by computing dose at the $8^{\circ}$ interspaced MSs. For this, the MUs of each step were equally split and attributed to the two defining MSs. Thereby, the number of MUs assigned to the first and last 
MS is the number of MUs of the arc divided by twice the number of steps. The MUs assigned to the other MSs is the number of MUs of the arc divided by the number of steps. Except where explicitly mentioned, all presented results on IMAT calculated dose distributions are based on this $8^{\circ}$ discretization.

A last MU optimization per arc was done using the results of the final dose computation. Finally, a prescription file was generated for each arc, and networked to an Elekta SLiPlus 18-MV linear accelerator. The IMAT treatment was delivered in local service mode using prototype dynamic software (Javelin, Elekta), with the same interlock class as used in clinical mode.

\section{Conventional planning}

The conventional plan consisted of two lateral beams and one posterior beam, as described earlier. The outlines of the beams were shaped conformally around the PTV with a margin of $0.8 \mathrm{~cm}$, using multileaf collimation. All beams consisted of a wedged and an unwedged part. The weight optimization was done using constrained matrix inversion (CMI) (16). It was proven previously that CMI resulted in a better plan than achieved by human planners, given that all relevant structures are included in the objective function. The following structures were used in the optimization algorithm: PTV, SB, and surr. The last structure was added to avoid unacceptable overdosage outside the PTV.

\section{Treatment evaluation}

Treatment delivery time, defined as the time between the start of the first arc and the end of the last arc, was measured for all patients for nearly all fractions. Apart from these clinical time measurements, dummy run delivery time measurements were done for all plans. For the IMAT plans, this was done on Javelin, whereas for the 3D plans, this was done on the RT Desktop (RTD) linac control software from Elekta. Total time was measured, as well as beam-on time, time for automatic setup, and time for system checks. For the different planning techniques, the total number of MUs needed to deliver a $180 \mathrm{cGy}$ fraction were also compared.

For dose-volume histogram (DVH) analysis, all plans were normalized to a median dose of 45 Gy in the PTV. Target dose homogeneity was evaluated using an inhomogeneity factor $U=\frac{\mathrm{D}_{99}-\mathrm{D}_{1}}{\mathrm{D}_{\text {med }}}$ with $D_{99}$ the dose below which 99\% of the PTV volume was treated, $D_{1}$ the dose below which $1 \%$ of the PTV volume was treated, and $D_{\text {med }}$ the median PTV dose. Furthermore, the $D_{99}$ and $D_{1}$ (used as a surrogate for maximum and minimum dose, respectively), and the $\%$ volume of the target structure receiving more than $95 \%$ of the prescribed dose over the total volume $\left(\mathrm{V}_{95 \%}\right)$ were compared. For SB, the mean dose $\left(D_{\text {mean }}\right)$, the $\%$ volume receiving more than $90 \%$ of the target prescription dose $\left(V_{90 \%}\right)$, and the \% volume receiving more than $15 \mathrm{~Gy}$ $\left(V_{>15 \mathrm{~Gy}}\right)$ were evaluated. Finally, the integral dose $\left(D_{I_{\text {totat }}}\right)$ given to the patient was approximated as $D_{\text {mean }} \times V_{\text {tot }}$ with $V_{\text {tot }}$ the total scanned volume. The dose deposition efficiency factor
(DDEF) was calculated by dividing the integral dose to the PTV $\left(D_{I_{P T V}}\right)$ by $D_{I_{\text {total }}}$. DVHs were recalculated for the group of 7 patients. For all relevant structures of all patients, the volume percentiles at every $0.25 \mathrm{~Gy}$ dose level from 0 to 50 Gy were computed from the calculated dose distribution. For every dose level, the mean and standard error of the mean (SEM) was calculated. In order to characterize the different plans in their ability to spare SB, dose "volume difference" histograms (DVDH) were made. A DVDH represents the difference (between two plans) of the \% volume receiving specified doses. DVDHs were constructed by calculating the mean (over the 7 patients) of difference in $\%$ volume receiving a specified dose. These points were calculated every 0.25 Gy between 0 and 50 Gy. For all statistical comparisons, a paired Student $t$ test was used. All tests were two-tailed, and $p<0.05$ was considered statistically significant.

\section{Dosimetric verification}

Polymer gel dosimetry (PGD) was used for dose verification. The basis of this dosimetric technique resides in a polymerization reaction of acrylic monomers within a hydrogel. The reaction is initiated by highly reactive radicals that are formed by radiolysis during irradiation. The amount of polymer formed is related to the absorbed dose, and can be quantified by MRI. Detailed information on PGD can be found elsewhere (17-19). A homogeneity study was done to validate PGD for the use of large volumes, and is described elsewhere (20). A Barex (Cifra, Chateau Thierry, France) cast was vacuum molded on the pelvic region of the RANDO phantom (Alderson Research Laboratories, Stamford, CT). Three supplemental RANDO slices were placed cranially and caudally to the Barex phantom to have full scatter conditions in the gel during irradiation. CT scans (Siemens Somatom Plus 4, Erlangen, Germany) of the gelfilled Barex phantom were transferred to the planning system, and the volumes of interest of a patient case were transferred to the CT set. For this setup, a 3D and an IMAT plan were made as described earlier, and a prescription was made giving a median dose of 7.5 Gy to the PTV. This ensured the maximal response range of the gel, while avoiding saturation effects in the gel. Both techniques were dosimetrically verified using PGD. For the IMAT plan, two measurements were done. First, the plan was delivered in arc therapy mode, thus with dynamic gantry $\left(\mathrm{IMAT}_{\mathrm{d}}\right)$. In a second experiment, the prescription was delivered in a discrete way, i.e., as a static gantry IMRT with gantry positions every $8^{\circ}\left(\mathrm{IMAT}_{\mathrm{s}}\right)$. The latter delivery reflects how the plan is calculated. By comparing both results, the effects of the interpolation from discrete, $8^{\circ}$ interspaced, gantry positions to arcs can be seen. For each experiment, gel-filled test tubes were irradiated to doses between 0 and 10 Gy (every $1 \mathrm{Gy)}$ for calibration purposes. The dose distributions, as measured in the gel, were transferred to the planning system. For the transfer of the gel-measured dose distributions, the following positioning procedure was followed: on both the planning CT and the MRI (used for PGD), the content of 
Table 2. Summary of the DVH data, showing the mean \pm standard deviation

\begin{tabular}{lccc}
\hline & 3D & IMAT & $p$ Value \\
\hline PTV & & & \\
$\mathrm{V}_{95}(\%)$ & $97.5 \pm 1.4$ & $95.7 \pm 2.0$ & 0.16 \\
$\mathrm{~V}_{107}(\%)$ & $0.0 \pm 0.0$ & $0.2 \pm 0.3$ & 0.13 \\
$\mathrm{D}_{99}(\mathrm{~Gy})$ & $46.6 \pm 0.1$ & $47.3 \pm 0.5$ & $<0.01^{*}$ \\
$\mathrm{D}_{1}(\mathrm{~Gy})$ & $42.1 \pm 0.6$ & $40.9 \pm 1.3$ & 0.12 \\
$\mathrm{U}(\%)$ & $9.9 \pm 1.5$ & $14.1 \pm 3.1$ & $0.03^{*}$ \\
Small bowel & & & \\
$\mathrm{D}_{\text {mean }}(\mathrm{Gy})$ & $17.0 \pm 7.7$ & $12.4 \pm 4.6$ & $0.02^{*}$ \\
$\mathrm{~V}_{90}(\%)$ & $19.1 \pm 6.5$ & $6.6 \pm 2.3$ & $0.04^{*}$ \\
$\mathrm{~V}_{>15 \mathrm{~Gy}}(\%)$ & $45.6 \pm 8.2$ & $33.0 \pm 5.6$ & $0.04^{*}$ \\
$\mathrm{Bladder}$ & & & \\
$\mathrm{D}_{\text {mean }}(\mathrm{Gy})$ & $34.6 \pm 6.8$ & $18.2 \pm 2.7$ & $<0.01^{*}$ \\
$\mathrm{D}_{\mathrm{I}_{\text {total }}}(\mathrm{J})$ & $262.1 \pm 64.5$ & $244.0 \pm 47.6$ & 0.24 \\
$\mathrm{DDEF}(\%)$ & $20.7 \pm 0.1$ & $22.2 \pm 0.1$ & 0.21 \\
\hline
\end{tabular}

Abbreviations: $3 \mathrm{D}=$ conventional plan (three beam directions), with conformal portals; IMAT $=$ intensity-modulated arc therapy plan; PTV = planning target volume; $V_{90}, V_{95}$, and $V_{107}=$ partial volume $(\%)$ receiving more than $90 \%, 95 \%$, and $107 \%$ of the prescribed dose, respectively; $D_{1}$ and $D_{99}=$ the first and the 99th percentile dose; $U=$ inhomogeneity factor, defined as $\frac{\left(D_{99}-D_{1}\right)}{D_{\text {med }}}$, with $D_{\text {med }}$ the median dose; $D_{\text {mean }}=$ mean dose; $D_{I_{\text {total }}}=$ the integral dose defined as the mean dose in the total volume multiplied by its volume, $\left(D_{I_{\text {total }}}\right.$ was calculated for the total treatment dose, $45 \mathrm{~Gy}$ ); DDEF = dose deposition efficiency factor, defined as $\mathrm{D}_{\mathrm{I}_{\mathrm{PTV}}} / \mathrm{D}_{\mathrm{I}_{\mathrm{total}}} ; p$ values represent the result of a two-tailed paired $t$ test.

* Statistically significant.

the Barex cast (= the gel) was automatically contoured. The center of volume (COV) of both structures was positioned onto each other. Rotations were avoided by using laser line positioning on the CT scanner and positioning lines on the MRI scanner. The measured dose distribution was converted to the prescription dose based on the planned vs. delivered MUs. As a consequence, the comparison of converted dose distributions is an absolute dosimetry comparison. The DVHs of all relevant structures, which were clipped to the volume of the gel phantom (11), were computed both for the calculated and the measured dose, and compared. Low's $\gamma$-index (21) was calculated in 3D (dose difference criterion $=5 \%$, distance to agreement $=5 \mathrm{~mm}$ ), and $\gamma$-volume histograms $(\gamma-\mathrm{VH})$ were reconstructed.

\section{RESULTS}

\section{Treatment plan and delivery}

Details on the delivered IMAT plans are summarized in Table 1 Three to six arcs were used in the IMAT plans. Mean clinical delivery time was 6.3 min (range, 3.2-12.8 min) over all fractions and all patients. For the dummy run delivery, the mean total delivery time was $5.5 \mathrm{~min}$ (range, 3.5-7.6 min) for the IMAT plans, and 2.5 min (range, 2.3-2.8 min) for the 3D plans $(p<0.01)$. When considering beam-on time only, this was $3.8 \pm 1.0$ min for IMAT and

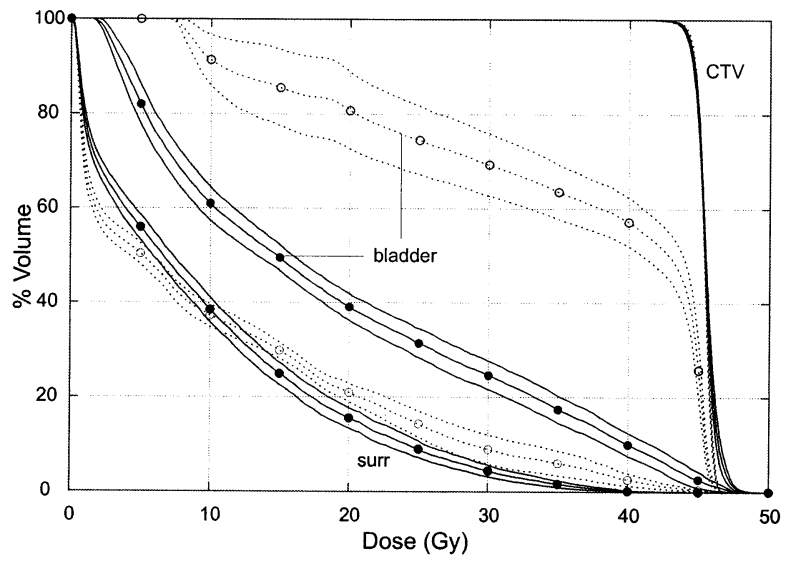

(a)

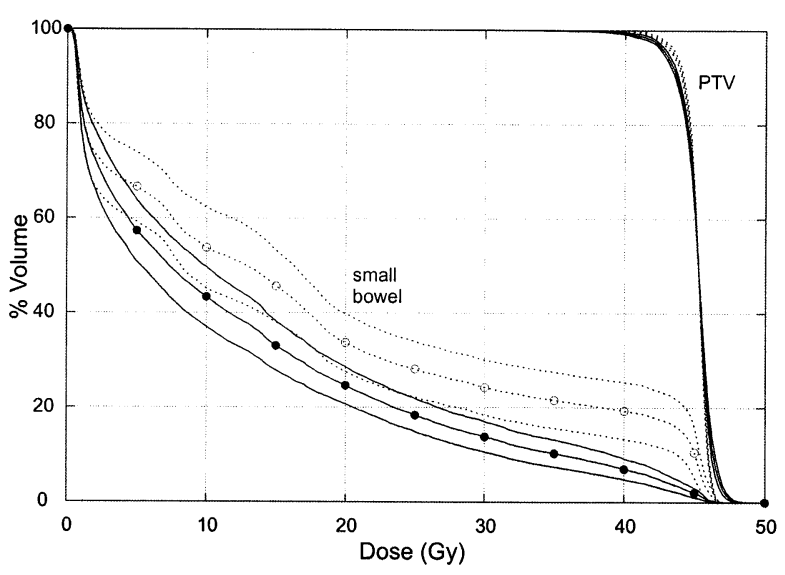

(b)

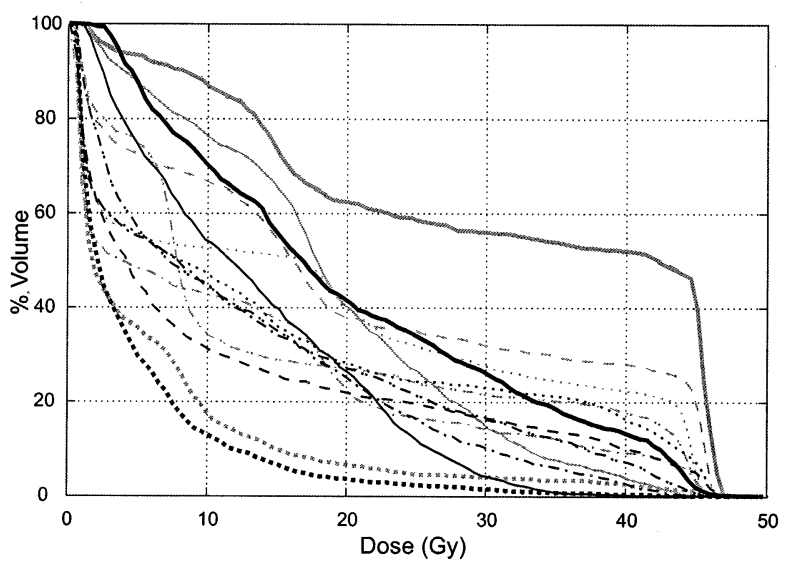

(c)

Fig. 3. Dose volume histograms (DVHs) compiled from the data of the whole group. Solid lines and black-filled circles represent the IMAT plans; the 3D plans are shown by the dotted lines and open circles. For reasons of clarity, all circles were omitted for CTV and PTV. For each plan and each structure, the mean \pm the standard error of the mean is represented. Data are shown for CTV, bladder, and surr (a), and PTV and small bowel (b). In (c), the individual DVHs for the 7 patients are shown, both for the 3D plans (gray lines) and for the IMAT plans (black lines). The DVHs for Patient 1 (solid lines) and for Patient 4 (dotted lines) are displayed in bold. 


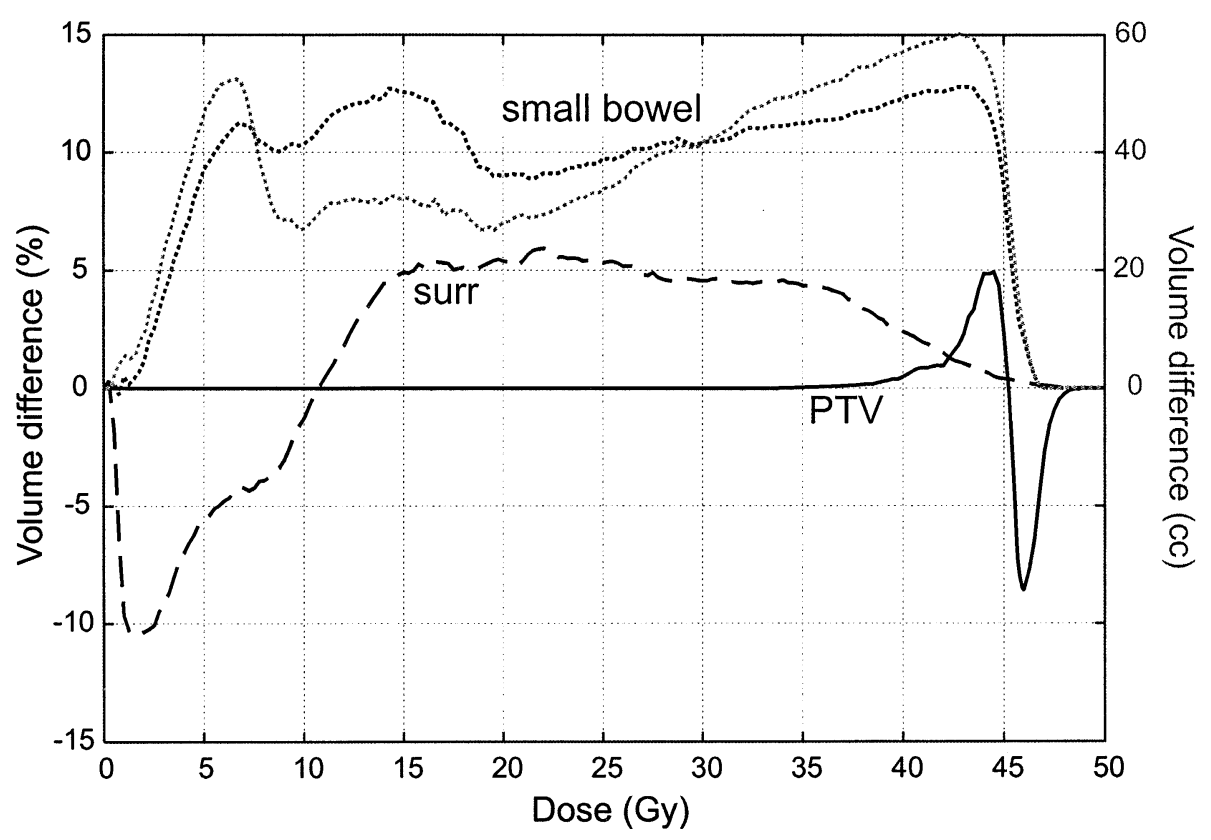

Fig. 4. Dose volume difference histograms (DVDH) for planning target volume (PTV) (solid line), small bowel (dotted line), and surr (dashed line). The gray dotted line represents the dose absolute volume difference histogram (DAVDH) for the small bowel (in cc). The $\mathrm{D}(\mathrm{A}) \mathrm{VDH}$ is constructed by plotting the mean (over the 7 patients) of $\left(\mathrm{V}_{\mathrm{X}_{3 \mathrm{D}}}-\mathrm{V}_{\mathrm{X}_{\mathrm{IMAT}}}\right)$, in which $\mathrm{V}_{\mathrm{X}}$ is the $\%$ volume (or the absolute volume for DAVDH) receiving dose $\mathrm{x}$. Points were calculated for $0 \mathrm{~Gy}$ $<\mathrm{x}<50$ Gy with an 0.25 Gy increment of $\mathrm{x}$. Positive values indicate that the volume irradiated to dose $\mathrm{x}$ was larger in the $3 \mathrm{D}$ plans than in the IMAT plans.

$1.4 \pm 0.2 \mathrm{~min}$ for the 3D delivery $(p<0.01)$. Time for system checks was $1.3 \mathrm{~min}$ for the IMAT delivery on the Javelin, and $0.4 \mathrm{~min}$ for the 3D plans on the RTD. For the IMAT plans, the number of MUs needed for a fraction of 180 cGy varied between 337 and 529 MUs (mean, $456 \pm 74$ MUs). For the 3D plans, this varied between 340 and 498 MUs (mean, $412 \pm 56$ ). This difference was not significant $(p=0.16)$.

\section{DVH analysis and dose distributions}

The compiled DVH data for the 7 patients are summarized in Table 2, and graphically displayed in Fig. 3. The minimal dose in the PTV was not significantly different. Inhomogeneity, however, was lower for the 3D plans $(9.9 \%$ $\pm 1.5)$ than for the IMAT plans $(14.4 \% \pm 3.1)$. This difference was significant $(p=0.03)$. Maximal dose in the PTV was 46.6 Gy for the 3D plans and $47.3 \mathrm{~Gy}$ for the IMAT plans $(p<0.01)$. For the CTV, the inhomogeneity for the 3D and IMAT plans was $6.3 \%$ and $8.1 \%(p=0.06)$. The mesorectal space, surrounding the rectum, received a slightly higher dose in the IMAT plans than in the 3D plans, with a maximal dose of 47.0 Gy vs. $46.3 \mathrm{~Gy}(p=0.01)$. In the $3 \mathrm{D}$ plans, no part of the mesorectal space received a dose higher than $107 \%$ of the prescribed dose, while the $V_{107}$ was $0.1 \%$ for the IMAT plans. This difference was not significant $(p=0.1)$. For $\mathrm{SB}$, the $D_{99}$ was $45.5 \mathrm{~Gy}$ for the 3D plans and $41.5 \mathrm{~Gy}$ for the IMAT plans. Mean dose to the SB was significantly lower for the IMAT plans, as was the $V_{>15 \mathrm{~Gy}}$ and the $V_{90}$. The absolute volume of SB receiving more than 15 Gy was $158 \mathrm{cc}$ (range, 21-327 cc) in the 3D plans, and $128 \mathrm{cc}$ (range, 13-305 cc) in the IMAT plans. The absolute $V_{90}$ was $84 \mathrm{cc}$ (range, 1-234 cc) and $27 \mathrm{cc}$ (range, $0-58 \mathrm{cc}$ ) for the 3D and IMAT plans, respectively. Figure 4 shows the DVDH for SB, PTV and surr for 3D plans compared to the IMAT plans. This illustrates that the volume of SB receiving any dose is lower for the IMAT plans. The differences in relative volume between 3D and IMAT plans for SB were significant between 13.5 and 42.5 Gy. For the PTV, the DVDH shows the underdosage and overdosage caused by IMAT when compared to 3D. Between 42.25 and $45 \mathrm{~Gy}, 1-5 \%$ of the volume of the PTV received less by IMAT than by $3 \mathrm{D}$. The bladder received a significantly lower dose in the IMAT plans, with a relative reduction of nearly $50 \%$ in $\mathrm{D}_{\text {mean }}$. The dose to the surr was lowest for the IMAT plans, with a mean $V_{>30 \mathrm{~Gy}}$ of $9.0 \%$ and $4.4 \%$ for the 3D and IMAT plan, respectively. This is also reflected in the average DDEF, which was $20.7 \%$ for the 3D plans and $22.2 \%$ for the IMAT plans. This difference was not significant, but shows that IMAT is at least as efficient as the 3D technique in dose deposition in the PTV.

Dose distributions for both an IMAT and a 3D plan are shown in Fig. 5. The concave sparing of the SB by the IMAT plan is clearly visible in the three planes. The isodose lines are highly conformal to the PTV form for the IMAT plan. The dose outside the PTV is distributed over a large volume in the IMAT plan, which can also be derived from the DVDH (Fig. 4), which shows that IMAT results in deposition of low dose (between 0 and 11 Gy) in a larger 


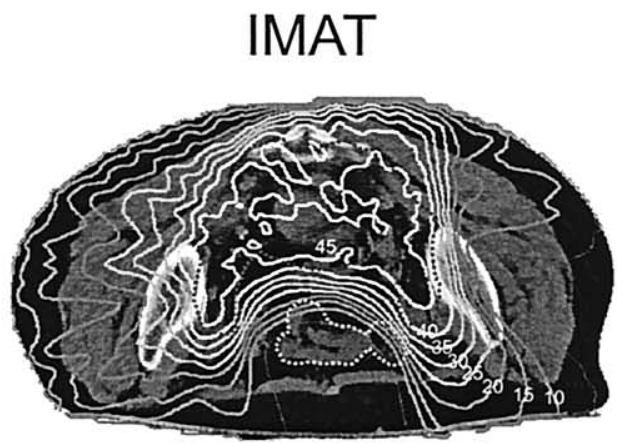

(a)

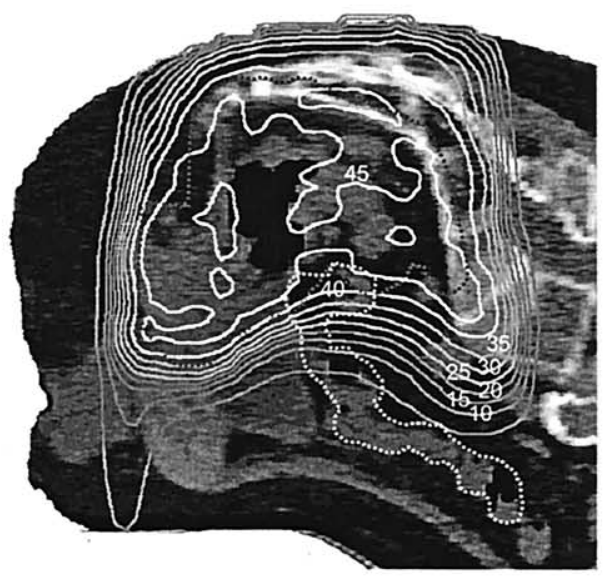

(c)

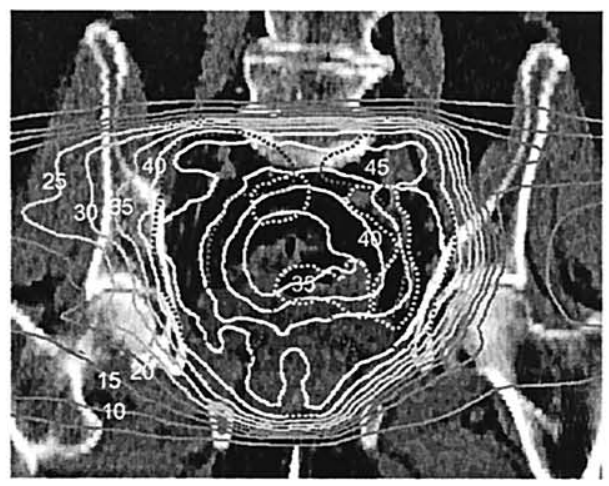

(e)

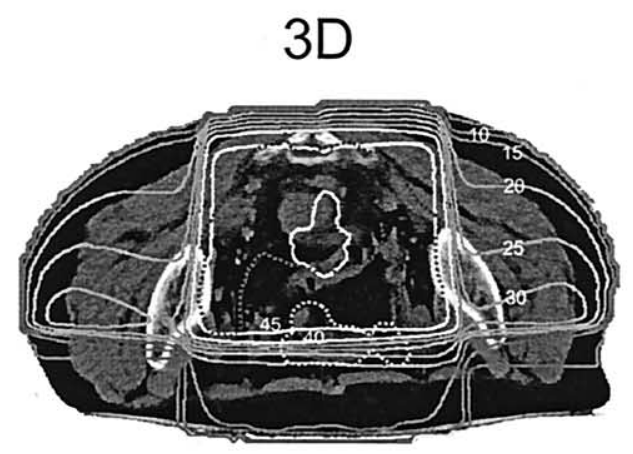

(b)

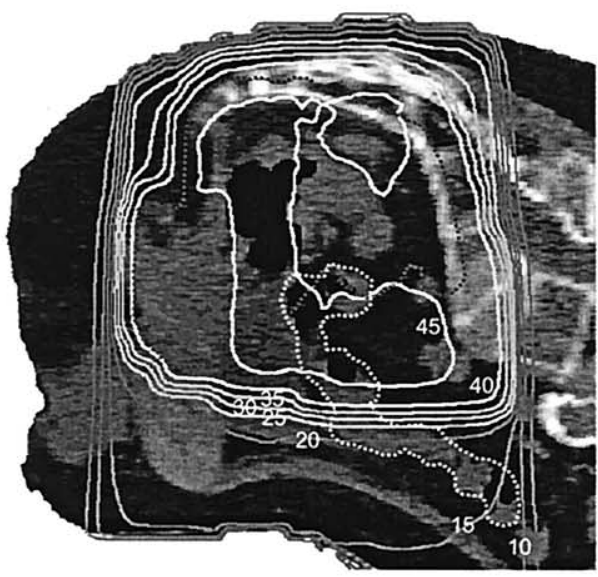

(d)

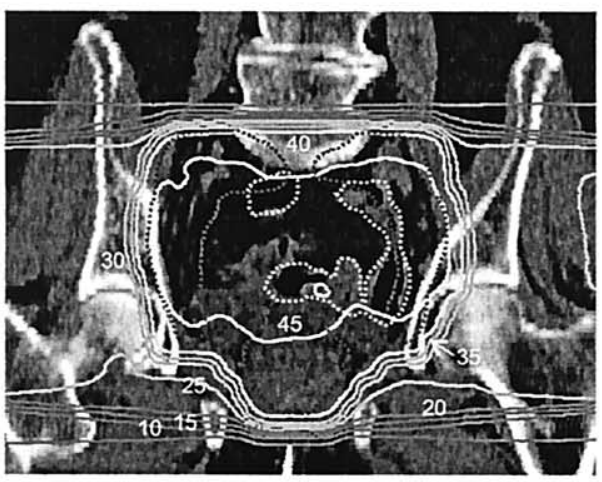

(f)

Fig. 5. Dose distributions for Patient 5, showing the delivered intensity-modulated arc therapy (IMAT) plan in a transverse (a), sagittal (c), and coronal (e) plane. The 3D plans (b, d, f) are shown in the same three planes. Isodose values are in Gy. The planning target volume is delineated by a dark dotted line, small bowel is shown by a light dotted line.

volume of the surr. The IMAT plan shows serrated isodose lines (Fig. 5a), especially in the low-dose region, caused by the discrete gantry angle calculations.

\section{Dosimetric verification}

The results of the PGD are summarized in Table 3 and in Figs. 6 and 7. Table 3 shows the volumes of the clipped structures, and the relative differences between the mea- sured and the calculated values for some clinically important parameters. The relative differences in $\mathrm{D}_{\text {med }}$ were insignificant, and varied between $-1.1 \%$ for the $3 \mathrm{D}$ plan and $0.3 \%$ for the $\operatorname{IMAT}_{d}$ experiment. The relative difference in maximal PTV dose $\left(D_{99}\right)$ was nowhere larger than $4 \%$, with the largest difference found for $\operatorname{IMAT}_{\mathrm{s}}(+4.0 \%)$. For all techniques, the $D_{99}$ was underestimated by the dose calculation algorithm, while the minimal dose $\left(D_{1}\right)$ was overes- 
Table 3. Summary of the results of the polymer gel dosimetry

\begin{tabular}{|c|c|c|c|c|}
\hline & $\begin{array}{c}\mathrm{Vol}_{\text {trunc }} \\
\text { (cc) }\end{array}$ & $3 \mathrm{D}$ & $\mathrm{IMAT}_{\mathrm{s}}$ & $\operatorname{IMAT}_{\mathrm{d}}$ \\
\hline PTV & $952(97 \%)$ & & & \\
\hline$\Delta \mathrm{D}_{\text {med }}(\%)$ & & -1.1 & -0.3 & +0.3 \\
\hline$\Delta \mathrm{D}_{99}(\%)$ & & +2.1 & +4.0 & +3.2 \\
\hline$\Delta \mathrm{D}_{1}(\%)$ & & +0.3 & -2.8 & -2.4 \\
\hline $\mathrm{Vol}_{\gamma>1}(\%)$ & & 0.0 & 1.6 & 1.1 \\
\hline Small bowel & $427(82 \%)$ & & & \\
\hline$\Delta \mathrm{D}_{\text {mean }}(\%)$ & & +5.6 & -0.5 & -0.5 \\
\hline$\Delta \mathrm{V}_{90 \%}(\%)$ & & +1.3 & -1.8 & -2.1 \\
\hline$\Delta \mathrm{V}_{>15} \mathrm{~Gy}(\%)$ & & +8.4 & +3.9 & +0.1 \\
\hline $\mathrm{Vol}_{\gamma>1}(\%)$ & & 1.6 & 6.1 & 9.7 \\
\hline Bladder & $124(100 \%)$ & & & \\
\hline$\Delta \mathrm{D}_{\text {mean }}(\%)$ & & +3.1 & +12.0 & +9.9 \\
\hline $\mathrm{Vol}_{\gamma>1}(\%)$ & & 0.0 & 6.0 & 11.8 \\
\hline surr & $6298(36 \%)$ & & & \\
\hline $\mathrm{Vol}_{\gamma>1}(\%)$ & & 11.7 & 9.4 & 5.3 \\
\hline Scanned volume & & & & \\
\hline $\mathrm{Vol}_{\gamma>1}(\%)$ & & 9.0 & 10.2 & 6.4 \\
\hline
\end{tabular}

Abbreviations: $\mathrm{PTV}=$ planning target volume; $\mathrm{Vol}_{\text {trunc }}=$ the volume of the structure, clipped to the volume of the MRImeasured gel phantom (The percentage between brackets is the partial volume of the structure in the gel phantom); IMATs = the IMAT treatment, delivered to the gel phantom in a static gantry mode; $\mathrm{IMAT}_{\mathrm{d}}=$ the IMAT treatment, delivered to the gel phantom in dynamic gantry mode; $\Delta \mathrm{D}_{\text {med }}=$ relative difference between measured and calculated mean dose, calculated as $\frac{D_{\text {med }_{\text {gel }}}-D_{\text {med }_{\text {calc }}}}{D_{\text {med }_{\text {calc }}}}$, with $\mathrm{D}_{\text {med }_{\text {gel }}}$ the median dose in the specified organ, as measured by gel dosimetry, and $\mathrm{D}_{\text {med }_{\text {calc }}}$ the median dose as calculated (The same reasoning was followed for $\Delta \mathrm{D}_{99}, \Delta \mathrm{D}_{1}$, $\Delta \mathrm{D}_{\text {mean }}, \Delta \mathrm{V}_{90 \%}$, and $\left.\Delta \mathrm{V}_{>15} \mathrm{~Gy}\right) ; \mathrm{Vol}_{\gamma>1}=$ the partial volume of the clipped structure in which the $\gamma$-index was higher than unity.

timated by $\pm 2.5 \%$ in IMAT, irrespective of the delivery (static vs. dynamic gantry). The partial volume of the PTV, in which the $\gamma$-index was higher than $1\left(\mathrm{~V}_{\gamma>1}\right)$, was nowhere higher than $2 \%$. No clear difference was found between the $\mathrm{IMAT}_{\mathrm{s}}$ and $\mathrm{IMAT}_{\mathrm{d}}$ results. For SB, the relative difference in $\mathrm{D}_{\text {mean }}$ was largest for the $3 \mathrm{D}$ experiment $(+5.6 \%)$, and smallest for both IMAT experiments $(<1 \%)$. The $\mathrm{V}_{\gamma>1}$ for SB was $9.7 \%$ for $\mathrm{IMAT}_{\mathrm{d}}$, although there is nearly no difference between calculated and measured dose when looking at the DVHs (Fig. 7a). The largest differences between measured and calculated dose were seen for bladder, for which the mean measured dose was up to $12 \%$ higher than the calculated dose (this was for $\mathrm{IMAT}_{\mathrm{s}}$ ). This is reflected in the high $\mathrm{V}_{\gamma>1}$ for $\operatorname{IMAT}_{\mathrm{s}}(6 \%)$ and $\mathrm{IMAT}_{\mathrm{d}}$ (11.8\%). The $\mathrm{V}_{\gamma>1}$ for the total scanned volume was lowest for the $\operatorname{IMAT}_{\mathrm{d}}(6.4 \%)$ and highest for the $\mathrm{IMAT}_{\mathrm{s}}(10.2 \%)$ delivery. On Fig. 7, the same data can be found. It shows the good correlation between the measured and calculated dose for PTV. It is also clear that there is no substantial difference between the IMAT $T_{s}$ and the $\mathrm{IMAT}_{\mathrm{d}}$ delivery. The DVHs for bladder show a higher measured than calculated dose in all cases, and this is most pronounced in the IMAT deliveries. For SB and surr, DVH comparison shows no clinically relevant differences. Calculated and gel-measured dose distributions are shown in Fig. 6. The ripples in the low-dose region, which are a consequence of the gantry angle discretization that is done for planning and calculations, are also obvious in the $\mathrm{IMAT}_{\mathrm{s}}$ measured dose distributions (Fig. 6c). They disappear when the dose computation is done for MSs, interspaced by $2^{\circ}$ instead of $8^{\circ}$ (Fig. $6 \mathrm{~b}$ ), which more closely resembles the results of the $\mathrm{IMAT}_{\mathrm{d}}$ delivery (Fig. 6d). This IMAT $\mathrm{T}_{\mathrm{d}}$ is the delivery mode used in the clinical IMAT execution.

\section{DISCUSSION}

Although not significant, the minimal dose was lower for the IMAT plans. The same can be seen for the $V_{95}$ of the PTV, which was lower for the IMAT plans. Still, on the DVH (Fig. 3b), it is clear that this underdosage is rather small. The PTV underdosage is partially caused by the SB sparing. The possibility to create concave dose distributions with IMAT leads to a larger PTV surface adjacent to the dose gradient. This, in combination with the leaf position optimization, can result in the observed underdosage, located at the borders of the PTV. Although the PTV underdosage in this group was deemed not to be clinically relevant, assigning a too high importance for SB in the optimization could result in relatively large dose "erosion" at the margins of the PTV. For this reason, we defined the following PTV acceptance criteria for future use in IMAT planning for rectal cancer, based on the results of the present analysis. For the following criteria, we took the observed mean $\pm 1 \mathrm{SD}$, thus resulting in (1) a $V_{95} \geq 93.7 \%$, (2) a $D_{1}$ $\geq 88 \%$ of the target prescription dose (for a prescription to $45 \mathrm{~Gy}$, this is $39.6 \mathrm{~Gy}$ ), and (3) a $V_{107} \leq 0.5 \%$. In case of a violation of one of the first two criteria, the IMAT plan should be reoptimized with lower SB importance. The third criterium should not be an absolute one, as the higher maximal dose (and more generally the higher inhomogeneity) is presumably caused by the discretization to deliverable arcs: ADR cannot be modulated in a fine sense due to the required constant ADR. Peaks visible in the ADR graphs cannot be expressed in the final arcs as the Elekta linac is not able to fluently deliver short arcs. Balancing the linac using counterweights and optimization of the settings of the gantry servo-system reduced the problems, but arcs smaller than $56^{\circ}$ still cause too many interrupts to be practical. The addition of a few static gantry intensity-modulated beams (replacing the practically undeliverable short arcs) to the IMAT arcs may reduce inhomogeneity in the PTV. This possibility has not yet been explored. During planning, it is clear that the arc extraction procedure significantly reduces the quality of the plan (PTV inhomogeneity and dose to the OARs increase, data not shown). This is (partly) solved by leaf position optimization. Earl et al. (22) also discuss the limitations imposed by the constant ADR, and showed that adding an additional arc could reduce this plan deterioration, but that a variable dose rate or gantry speed would solve this problem. We prefer the implementation of a variable gantry speed over the variable dose rate. In this 


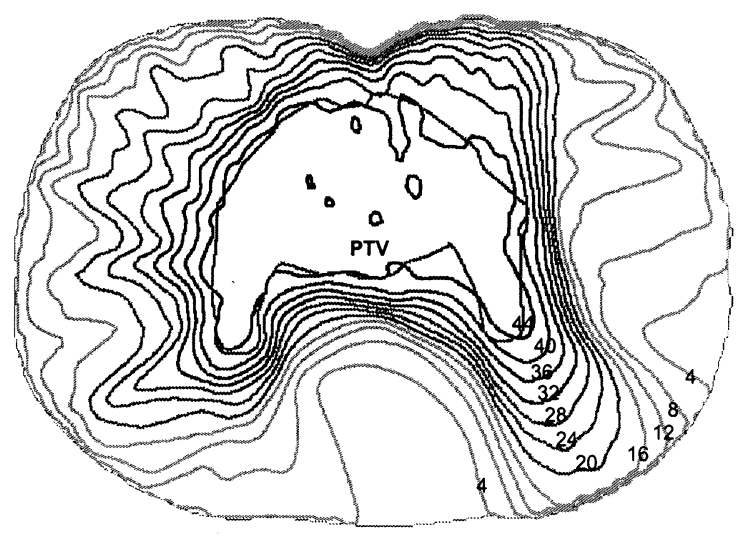

(a) calculated dose: 8 degrees interspacing

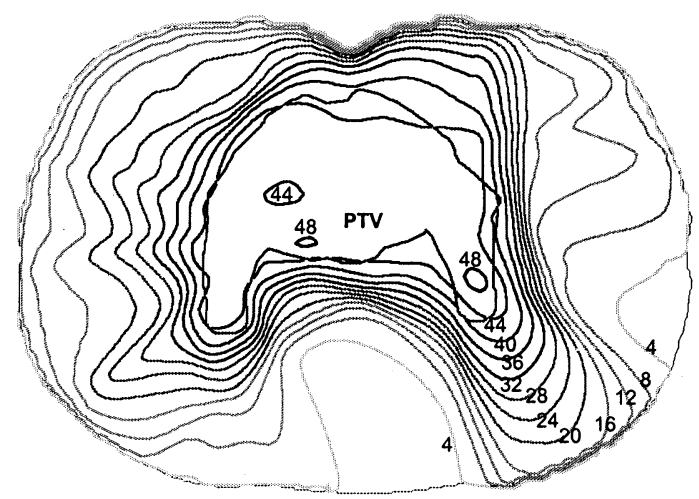

(b) calculated dose: 2 degrees interspacing

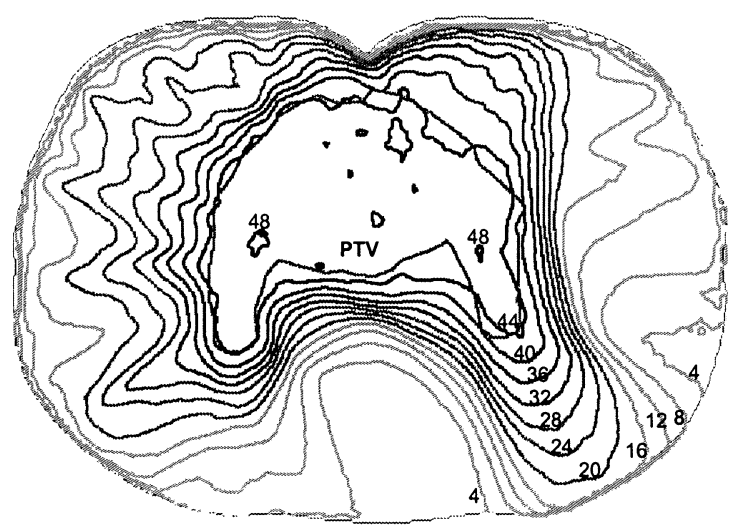

(c) measured dose: static gantry delivery

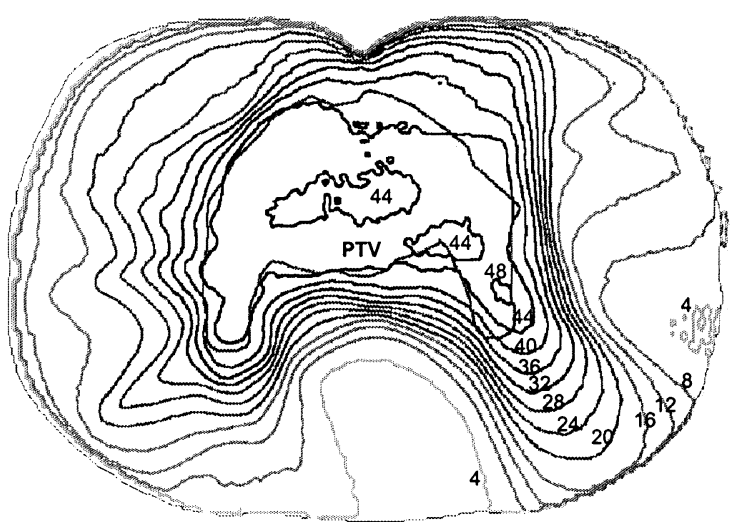

(d) measured dose: dynamic gantry delivery

Fig. 6. Dose distributions of the intensity-modulated arc therapy (IMAT) plan in a transverse plane through the isocenter as it was calculated (a, b) and measured by polymer gel dosimetry (c, d). Isodose values are in Gy. In (a), the calculations are shown for the IMAT plan, with calculated control points every $8^{\circ}$. In (b), the same plan is shown, but additional control points were generated and calculated every $2^{\circ}$ by interpolation of the preexisting control points. (c) The measured IMAT plan, delivered to the phantom in a discrete way, i.e. as a static gantry intensity-modulated radiotherapy with $8^{\circ}$ interspaced beams. (d) The measured IMAT plan, delivered in dynamic gantry mode.

way, the optimization even has the possibility to insert a static gantry IM beam as a part of an arc by lowering the gantry speed to $0 \% \mathrm{~min}$. This solution, which delivers the optimized nonconstant ADR, also reduces the number of arcs, the total gantry rotation, and most probably also the planning and treatment time. It also removes the complex planning step of arc extraction. We plan to investigate the full impact of variable ADR on planning quality and delivery time.

Intensity-modulated arc therapy offers the possibility to spare SB and bladder in comparison with a $3 \mathrm{D}$ technique. A relative reduction of $28 \%$ in $D_{\text {mean }}$ can be seen for SB. For the $V_{90}$, a relative reduction of $65 \%$ was reached by applying IMAT. The $V_{>15 \mathrm{G} y}$, which was found to be strongly associated with acute SB toxicity by Baglan et al. (6), was reduced by $28 \%$. Whether this sparing of the SB on the planning level will translate into a clinically diminished SB toxicity is beyond the scope of this study, which was focused on clinical implementation of IMAT and its planning comparison with a 3D technique. In the present series, the volume of SB receiving $90 \%$ of the target dose in the 3D plans $\left(\mathrm{Vol}_{\mathrm{sb}_{3 \mathrm{D}}}\right)$ was rather small (mean, $84 \mathrm{cc}$; range, 1-234 cc) compared to previous reports $(6,9)$. This might underestimate the full potential of IMAT, as we could expect a correlation between the $\mathrm{Vol}_{\mathrm{sb}_{3 \mathrm{D}}}$ and the relative reduction in $\mathrm{V}_{90}$, obtained by IMAT over 3D. A DVH analysis for the subgroup of patients with a $\mathrm{Vol}_{\mathrm{sb}_{3 \mathrm{D}}}$ greater than $84 \mathrm{cc}$ shows a $\mathrm{V}_{90}$ of $32.3 \%$ and $9.6 \%$ for 3D and IMAT, respectively. For the other 4 patients, the $\mathrm{V}_{90}$ was $9.2 \%$ and $4.4 \%$. Of course, the number of patients is too small to draw strong conclusions, but we hypothesize that the IMAT technique gets more efficient as the anatomic situation is more complex. Some subgroups of patients might have a large improvement of their treatment plan quality by IMAT, while the effect might be negligible or clinically irrelevant in others. It was shown that patients who receive postoperative radiotherapy have a larger portion of (fixed) SB in the pelvis (23). These patients are also at higher risk for SB toxicity 


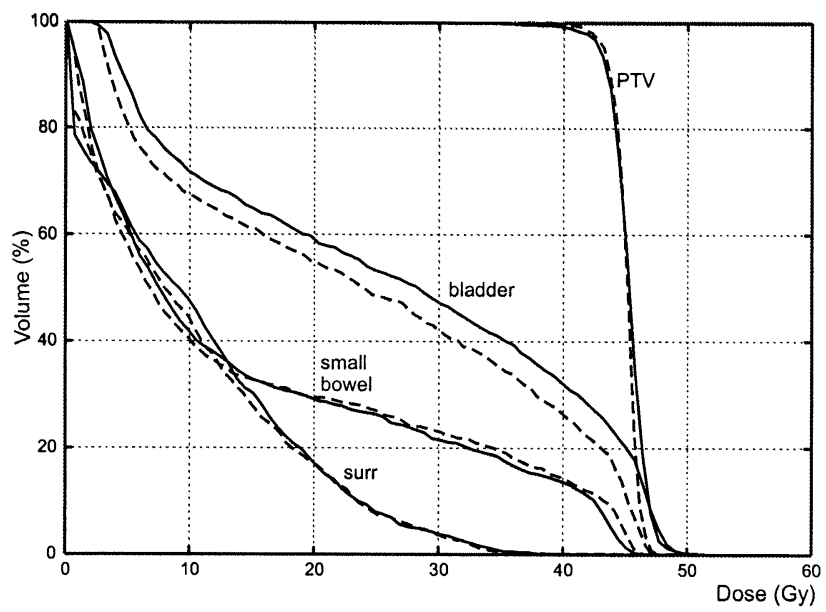

(a)

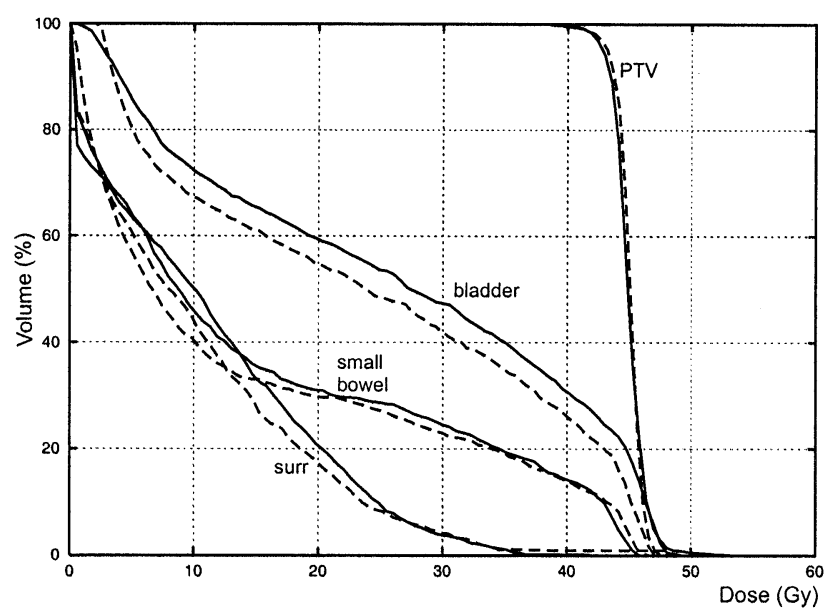

(c)

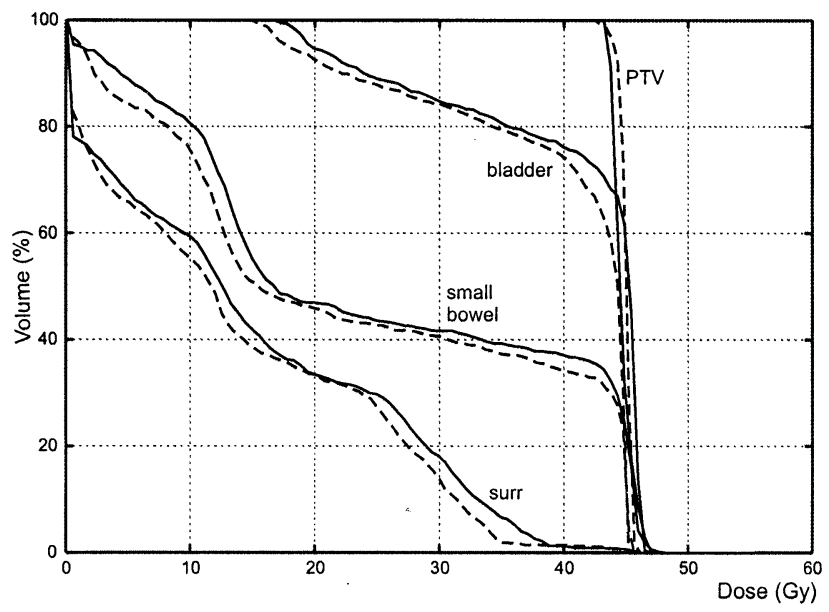

(e)

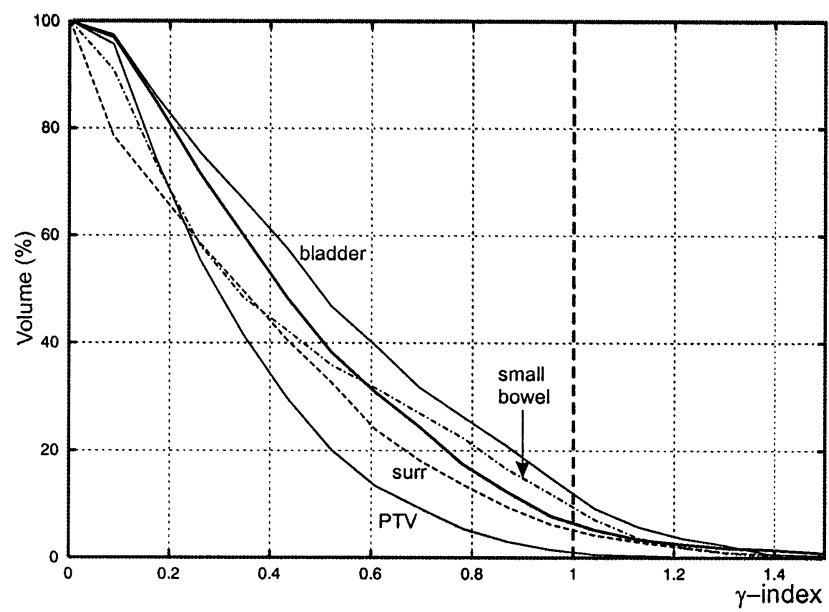

(b)

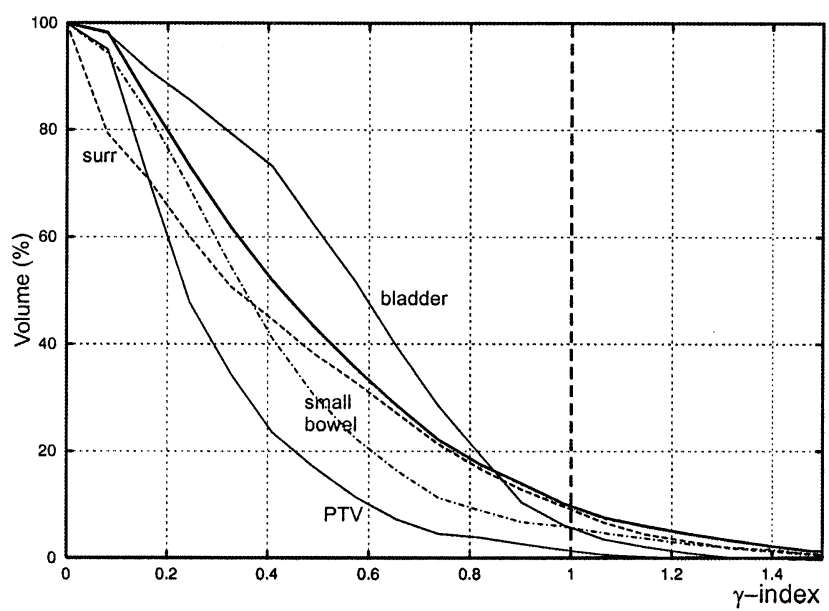

(d)

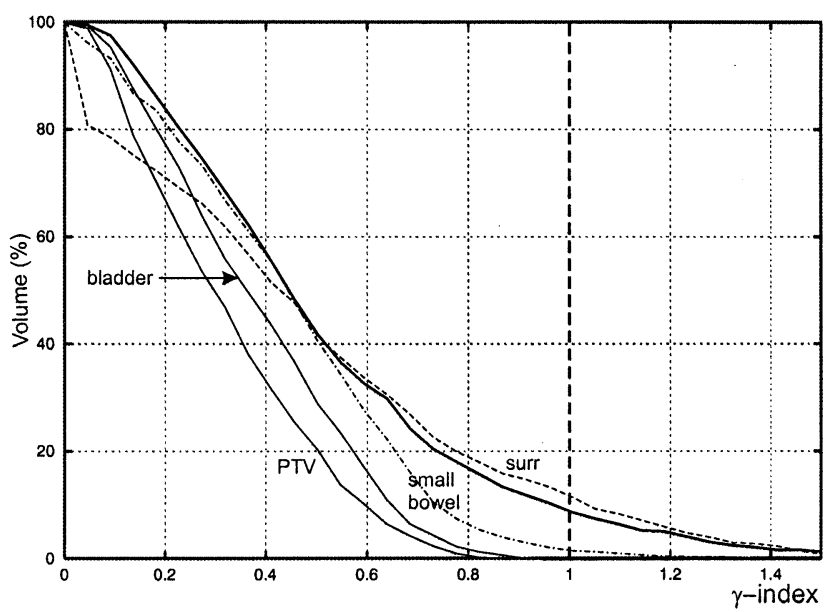

(f)

Fig. 7. Dose-volume histograms (DVHs) (a, c, e) and $\gamma$-volume histograms (b, d, f) for the three polymer gel experiments. In (a, c, e), the dashed lines represent the calculated DVHs, while the solid lines represent the gel-measured DVHs. The DVHs and $\gamma$-VHs are shown for planning target volume, small bowel, bladder, and surr. The $\gamma$-VHs for the total measured volume are also shown (bold curve). Results are shown for the dynamically delivered intensitymodulated arc therapy (IMAT) treatment $(a, b)$; the statically delivered IMAT plan $(\mathrm{c}, \mathrm{d})$, and for the 3D plan (e, f). 
than patients who received their radiotherapy in a preoperative setting (24). Besides this group of postoperatively treated patients, other patients who might benefit from IMAT could be selected by means of investigations such as volumetry of the contrast-filled SB during simulation.

The delivery time of the $3 \mathrm{D}$ treatments was significantly shorter than for the IMAT treatments. As mentioned, the 3D treatments were delivered using the RTD. The RTD system operates with a dose rate of $\pm 550 \mathrm{MUs} / \mathrm{min}$ (compared to 130 or $260 \mathrm{MUs} / \mathrm{min}$ for most of the arcs), and has a faster checking system than the prototype dynamic software used for IMAT. Thus, although clinical IMAT delivery time is within a standard time slot at the moment, it could be reduced even further by adaptations to the linac control software.

Quality assurance of a complex technique like IMAT remains a challenge. As both the gantry and the leaves are constantly moving, tracking their position is not easy. Ramsey et al. (25) developed an elegant method to evaluate the leaf position errors during arc delivery, based on the logs of a Varian Clinac MLC, and derived a formula to estimate the consequent dosimetrical error. Our strategy was to check the end-of-chain result. Different methods, such as ion chamber point dosimetry and 2D film dosimetry, have been used by others $(22,26)$. Although valuable, these techniques do not offer the possibility to evaluate the IMAT treatment in three dimensions, whereas dose distributions of IMAT treatment plans typically vary in three dimensions. PGD offers absolute dosimetry in three dimensions with a high resolution. Vergote et al. (20) optimized PGD for its use in large phantoms. This resulted in a structural root-mean-square deviation between gel-measured and Helax-TMS computed dose distributions of $2 \%$ (except in high dose gradient regions, where it was $5 \%$ ) and a stochastic deviation of $2 \%$, resulting in an accuracy of $2.8 \%$ (and $5.4 \%$ in high dose gradient regions). We found a good correlation between gel-measured and calculated dose distributions for the PTV $\left(\mathrm{V}_{\gamma>1}=1.1 \%\right)$. The SB sparing, obtained by the IMAT technique, was confirmed by the PGD measurements. These results ensured us that IMAT planning, calculation, and delivery were accurate and could be implemented in a clinical setting. The largest deviations were seen for bladder, with a calculated vs measured $\mathrm{D}_{\text {mean }}$ of $23.7 \mathrm{~Gy}$ and 26.0 Gy in the $\mathrm{IMAT}_{\mathrm{d}}$ experiment $(+9.9 \%)$. Nearly the full dose contribution to the bladder is due to scatter and transmission, as the bladder is shielded in all arc apertures. Calculation errors in the computation of both scatter dose and transmission can thus have a high impact on the calcu- lated dose of the bladder. PGD also showed that the interpolation of the $8^{\circ}$ angularly interspaced control points to an arc does not produce different dosimetrical results than if it were given as static gantry IM beams. As previously demonstrated $(20,26)$, decreasing the interspacing distance from $8^{\circ}$ to $2^{\circ}$ affects the dose distribution for the low-dose regions (the ripples disappear), whereas for high dose regions, there is little or no difference.

To our knowledge, there has been no report on clinically executed IMAT or even intensity-modulated radiotherapy (IMRT) treatment for rectal cancer until now, despite the fact that IMRT has been used in a clinical setting for nearly a decade now. There is one report of a planning comparison between IMRT and 3D (27). The authors found a relative reduction of $55 \%$ to $72 \%$ in $\mathrm{V}_{95}$ for $\mathrm{SB}$, depending on the PTV inhomogeneity that was tolerated. This is in the same range as our findings, with a $\mathrm{V}_{95}$ for SB $72 \%$ for IMAT. In pelvic radiotherapy, the first clinical results about the implementation of IMRT for gynecologic malignancies have been published. Mundt et al. have shown that IMRT reduces both acute (28) and chronic (29) SB toxicity in comparison to conventional treatment. In cervical cancer, as in rectal cancer, the internal iliac and obturator nodes are important routes of nodal spread, leading to a concave shaped PTV partially encompassing bladder and SB. Therefore, we hypothesize that a similar toxicity reduction can be achieved in rectal cancer patients as in patients with cervical cancer.

Whether IMAT will prove to be the best option for rectal cancer irradiation (and for other sites) in the future will largely depend on the improvement of IMAT. A large window for improvement exists for IMAT, including plan optimization and automation of the planning process, and improvements of the linac control software, of which variable gantry speed is the single most important.

\section{CONCLUSION}

Seven patients with rectal cancer were irradiated with IMAT. On planning, IMAT allowed one to spare SB as compared to a conventional 3D technique, without compromising the dose in the PTV. Although treatment time for IMAT was longer in comparison to a 3D technique, it was deliverable within a time slot of 5-10 min. Three-dimensional PGD showed that IMAT delivery is as accurate as 3D delivery. We identified significant potential for improvements both at the levels of planning and delivery. The single most important technical improvement for IMAT is the implementation of a variable gantry speed.

\section{REFERENCES}

1. Glimelius B. The role of radiotherapy in rectal cancer. Eur $J$ Cancer 2001;37(Suppl. 7):S203-212.

2. Colorectal Cancer Collaborative Group. Adjuvant radiotherapy for rectal cancer: A systematic overview of 8507 patients from 22 randomised trials. Lancet 2001;358:1291-1304.

3. Ahmad NR, Marks G, Mohiuddin M. High-dose preoperative radiation for cancer of the rectum: Impact of radiation dose on patterns of failure and survival. Int J Radiat Oncol Biol Phys 1993;27:773-778.

4. Chan AK, Wong AO, Langevin J, et al. Preoperative chemotherapy and pelvic radiation for tethered or fixed rectal cancer: A phase II dose escalation study. Int J Radiat Oncol Biol Phys 2000;48:843-856.

5. Kollmorgen CF, Meagher AP, Wolff BG, et al. The long-term 
effect of adjuvant postoperative chemoradiotherapy for rectal carcinoma on bowel function. Ann Surg 1994;220:676-682.

6. Baglan KL, Frazier RC, Yan D, et al. The dose-volume relationship of acute small bowel toxicity from concurrent 5-FU-based chemotherapy and radiation therapy for rectal cancer. Int J Radiat Oncol Biol Phys 2002;52:176-183.

7. Minsky B, Conti JA, Huang Y, et al. Relationship of acute gastrointestinal toxicity and the volume of irradiated small bowel in patients receiving combined modality therapy for rectal cancer. J Clin Oncol 1995;13:1409-1416.

8. Miller AR, Martenson JA, Nelson $\mathrm{H}$, et al. The incidence and clinical consequences of treatment-related bowel injury. Int $J$ Radiat Oncol Biol Phys 1999;43:817-825.

9. Letschert JG, Lebesque JV, Aleman BM, et al. The volume effect in radiation-related late small bowel complications: Results of a clinical study of the EORTC Radiotherapy Cooperative Group in patients treated for rectal carcinoma. $R a$ diother Oncol 1994;32:116-123.

10. Letschert JGJ. The prevention of radiation-induced small bowel complications. Eur J Cancer 1995;31:1361-1365.

11. Duthoy W, De Gersem W, Vergote K, et al. Whole abdominopelvic radiotherapy (WAPRT) using Intensity Modulated Arc Therapy (IMAT): First clinical experience. Int J Radiat Oncol Biol Phys 2003;58:1019-1032.

12. Yu C. Intensity-modulated arc therapy with dynamic multileaf collimation: An alternative to tomotherapy. Phys Med Biol 1995;40:1435-1449.

13. Nuyttens JJ, Robertson JM, Yan D, et al. The variability of the clinical target volume for rectal cancer due to internal organ motion during adjuvant treatment. Int J Radiat Oncol Biol Phys 2002;53:497-503.

14. De Neve W, Duthoy W, Claus F, et al. Dose conformation in IMRT for head and neck tumors: Which solution to apply? Cancer/Radiotherapie 2002;6(Suppl. 1):32-36.

15. De Gersem W, Claus F, De Wagter C, et al. An anatomybased beam segmentation tool for intensity-modulated radiation therapy and its application to head-and-neck cancer. Int $J$ Radiat Oncol Biol Phys 2001;51:849-859.

16. De Wagter C, Colle CO, Fortan LG, et al. 3D conformal intensity-modulated radiotherapy planning: Interactive optimization by constrained matrix inversion. Radiother Oncol 1998;47:69-76.

17. Maryanski MJ, Gore JC, Kennan RP, et al. NMR relaxation enhancement in gels polymerized and cross-linked by ionizing radiation: A new approach to 3D dosimetry by MRI. MRI 1993;11:253-258.

18. De Deene Y, De Wagter C, Van Duyse B, et al. Validation of MR-based polymer gel dosimetry as a preclinical three-dimensional verification tool in conformal radiotherapy. Magnet Reson Med 2000;43:116-125.

19. De Deene Y. Gel dosimetry for the dose verification of intensity-modulated radiotherapy treatments. Z Med Phys 2002;12: 77-88.

20. Vergote K, De Deene Y, Duthoy W, et al. Validation and application of polymer gel dosimetry for the dose verification of an intensity-modulated arc therapy (IMAT) treatment. Phys Med Biol 2004;49:287-305.

21. Low DA, Harms WB, Mutic S, et al. A technique for the quantitative evaluation of dose distributions. Med Phys 1998; 25:656-661.

22. Earl MA, Shepard DM, Naqvi S, et al. Inverse planning for intensity-modulated are therapy using direct aperture optimization. Phys Med Biol 2003;48:1075-1089.

23. Nuyttens JJ, Robertson J, Yan D, et al. The position and volume of the small bowel during adjuvant radiation therapy for rectal cancer. Int J Radiat Oncol Biol Phys 2001;51:12711280.

24. Minsky BD, Cohen AM, Kemeny N, et al. Combined modality therapy of rectal cancer: Decreased acute toxicity with the preoperative approach. J Clin Oncol 1992;10:1218-1224.

25. Ramsey CR, Spencer KM, Alhakeem R, et al. Leaf position error during conformal dynamic arc and intensity modulated arc treatments. Med Phys 2001;28:67-72.

26. Yu C, Li XA, Ma L, et al. Clinical implementation of intensity-modulated arc therapy. Int J Radiat Oncol Biol Phys 2002;53:453-463.

27. Robertson J, Yan D, Girimonte P, et al. The potential benefit of intensity modulated radiation therapy (IMRT) for rectal cancer (Abstr.). Int J Radiat Oncol Biol Phys 1999;45(Suppl. 3):248-249.

28. Mundt AJ, Lujan AE, Rotmensch J, et al. Intensity-modulated whole pelvic radiotherapy in women with gynecologic malignancies. Int J Radiat Oncol Biol Phys 2003;52:13301337.

29. Mundt AJ, Mell LK, Roeske JC. Preliminary analysis of chronic gastrointestinal toxicity in gynecology patients treated with intensity-modulated whole pelvic radiation therapy. Int $J$ Radiat Oncol Biol Phys 2003;56:1354-1360. 